\title{
Comunidades de esponjas do curso superior dos rios Paraná (Goiás) e Paraguai (Mato Grosso), Brasil, com redescrição de Oncosclera schubarti (Bonetto \& Ezcurra de Drago)
}

\author{
Twiggy Cristina Alves Batista ${ }^{1}$ \\ Cecília Volkmer-Ribeiro ${ }^{2}$
}

\begin{abstract}
Sponge communities of the upper rivers Paraná (Goiás) and Paraguai (Mato Grosso), Brazil, with redescription of Oncosclera schubarti (Bonetto \& Ezcurra de Drago). An extensive survey for freshwater sponges was carried at the temporarely exposed rocky bottoms of river Corumbá at Goiás State and river Manso at Mato Grosso State. The exposure resulted from the closing of the dams for lake formation at the respective Hidreletric Power Plants. Drulia uruguayensis Bonetto \& Ezcurra de Drago, 1968, Corvospongilla seckti Bonetto \& Ezcurra de Drago, 1966 and Trochospongilla repens (Hinde, 1888) were seen to be common to both rivers. Eunapius fragilis (Leidy, 1851), Oncosclera navicella (Carter, 1881) and $O$. petricola (Bonetto \& Ezcurra de Drago, 1969) were yet found in river Manso and O. schubarti (Bonetto \& Ezcurra de Drago, 1967) in river Corumbá. O. schubarti is redescribed and SEM illustrated.

KEY WORDS. Sponges, Oncosclera schubarti, Neotropical, river, benthic assemblages,
\end{abstract}

As únicas esponjas até agora registradas para as cabeceiras do Paraná, na região Centro-Oeste do Brasil, eram Sterrastrolepis brasiliensis Volkmer-Ribeiro \& De Rosa-Barbosa, 1978 no rio Turvo, contribuinte do Paranaíba na localidade de Paraúna (VOLKMER-Ribeiro \& ROSA-BARBOSA 1978) e, em ambiente lêntico, Metania spinata Carter, 1881 e Dosilia pydanieli Volkmer-Ribeiro, 1992, constituindo jazida de espongilito na lagoa do Alecrim, município de São Simão, à margem direita do rio Paranaíba (VOLKMER-RIBEIRO \& MOTTA 1995).

A possibilidade de amostrar extensivamente as comunidades de esponjas do leito profundo de grandes rios brasileiros surgiu, nas últimas décadas, com a intensificação da construção de barragens para formação dos lagos de hidrelétricas. Tais prospecções vem sendo feitas tanto no momento do desvio dos rios para a construção das barragens quanto por ocasião do fechamento das comportas para a formação dos lagos. Nesses momentos, os leitos à jusante das barragens ficam expostos, permitindo amostragens significativas das comunidades de esponjas porventura aí fixadas.

1) Secretaria de Estado da Saúde. Praça dos Girassóis, 77003-903 Palmas, Tocantins, Brasil. Bolsista do CNPq. E-mail: twiggybat@hotmail.com

2) Museu de Ciências Naturais, Fundação Zoobotânica do Rio Grande do Sul. Caixa Postal 1188, 90690-000 Porto Alegre, Rio Grande do Sul, Brasil. Pesquisadora do CNPq. E-mail: cvolkmer@fzb.org.br 
O fechamento das comportas da barragem da Usina Hidrelétrica (UHE) Corumbá, no rio Corumbá, bacia do Paraná, ocasionou a exposição temporária do leito à jusante, permitindo um primeiro levantamento da fauna de esponjas do bentos profundo desse rio, nos municípios de Corumbaíba e Ipamerí (Goiás). A formação do lago da UHE propiciou, por sua vez, acesso fácil, por barco, às cabeceiras ainda não afogadas dos córregos situados à montante da barragem, permitindo o resgate científico da fauna de esponjas nesses locais e levando à previsão das espécies eventuais colonizadoras do lago. As amostras estudadas possibilitaram estabelecer comparação com levantamentos feitos por BONETTO \& EZCURRA DE DRAGO (1967) para essa fauna, no curso médio do rio Paraná, Argentina.

$\mathrm{O}$ fechamento das comportas da barragem da UHE Manso permitiu a coleta no leito exposto do rio, à jusante da barragem. O rio Manso é contribuinte do Cuiabá, que faz parte da bacia do Paraguai, constituindo-se aqui a primeira notícia de esponjas para essa Bacia no Brasil.

\section{MATERIAL E MÉTODOS}

\section{Descrição da área}

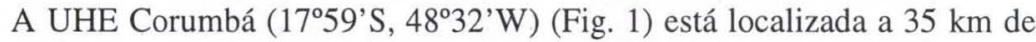
Caldas Novas, no estado de Goiás. A barragem represa o rio Corumbá e pequenos contribuintes à montante, numa área de $65 \mathrm{~km}^{2}$.

As coletas foram realizadas no leito do rio à jusante da barragem e em dois contribuintes da margem esquerda, à montante da mesma: córrego do Libório (Fig.

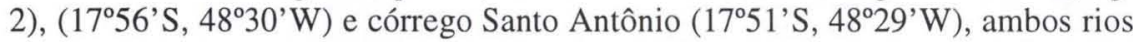
de primeira ordem, correntosos, com fundo constituído por rochas de grande porte, pedras menores e pouca areia. Nos locais de amostragem à jusante da barragem, no Rio Corumbá, (17 $\left.59^{\circ} \mathrm{S}, 48^{\circ} 32^{\prime} \mathrm{W}\right)$ o leito apresentava-se eminentemente arenoso e siltoso, com exposição de grandes blocos rochosos (Fig. 3) ou coberto por pedras roladas (Fig. 4), com faixas de areia marginais evidenciando assoreamento e mostrando os limites de cheia e seca do rio.

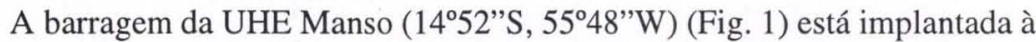
jusante da confluência dos rios Casca e Manso, a 90km de Cuiabá, no município de Chapada dos Guimarães, Mato Grosso. O lago da UHE cobre uma área de aproximadamente $428 \mathrm{~km}^{2}$.

As coletas foram realizadas à jusante da barragem, onde o leito, parcialmente exposto, mostrou fundo rochoso irregular contínuo (Fig. 5), com reduzida quantidade de areia.

\section{Métodos}

A prospecção no rio Corumbá foi realizada entre 30 de setembro e 05 de outubro de 1996 e no rio Manso entre 30 de novembro e 07 de dezembro de 1999. A amostragem, feita manualmente e a conservação dos materiais seguiram VOLKMERRIBEIRO (1985). As amostras foram catalogadas e depositadas na Coleção de Porifera do Museu de Ciências Naturais (MCN), Fundação Zoobotânica do Rio Grande do Sul (FZB). A preparação dos materiais para estudos e ilustrações ao microscópio óptico (câmara lúcida) seguiu VOLKMER-RIBEIRO (1985) e, ao Microscópio Eletrônico de Varredura (JEOL SM 5200), VOLKMER-RIBEIRO \& TURCQ (1996). Para a redescrição 
de $O$. schubarti foram realizadas mensurações de 50 megascleras e gemoscleras de cada espécime. Os dados abióticos apresentados para o córrego do Libório foram obtidos com aparelhos que compõem o laboratório portátil modelo F-1003: medidor de oxigênio (VDSF - Umwelttechnik, F-1001), de pH (VDSF - Umwelttechnik, F-1002) e de condutividade (VDSF - Umwelttechnik, F-1000).

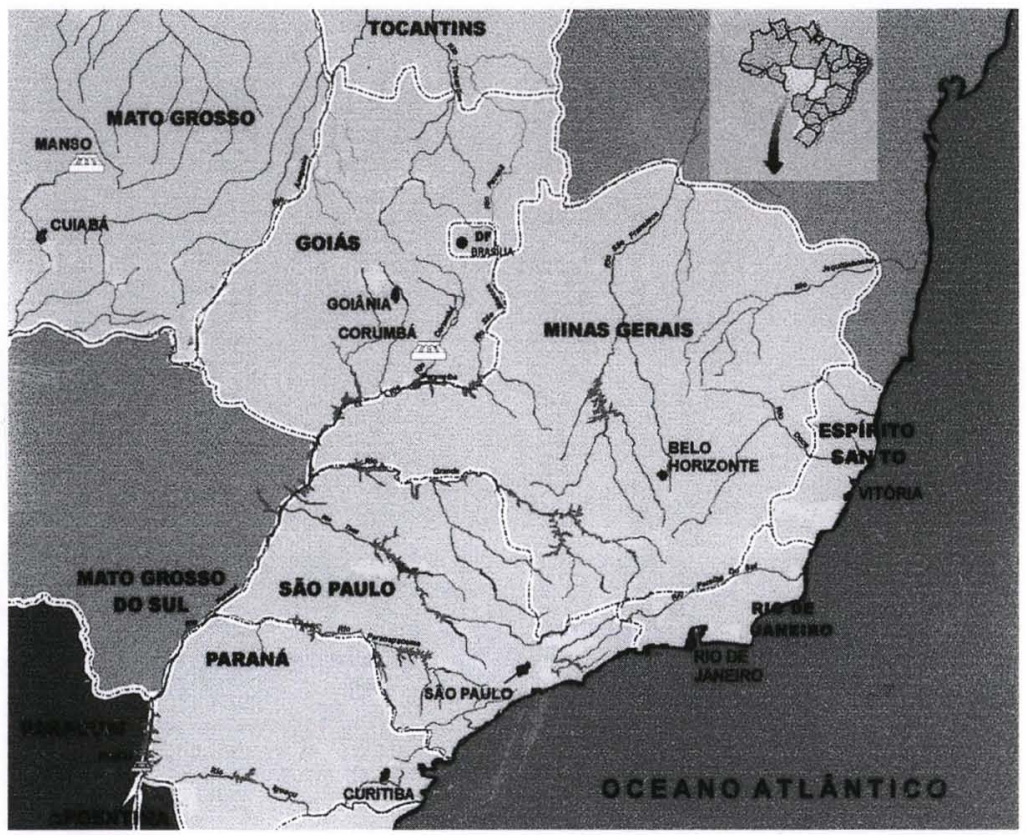

Fig. 1. Mapa da área de estudo assinalando as Usinas Hidrelétricas de Manso (Mato Grosso) e Corumbá (Goiás). Fonte: Furnas S.A.

\section{RESULTADOS}

\section{Potamolepidae Brien, 1967 Oncosclera Volkmer-Ribeiro, 1970}

\section{Oncosclera schubarti (Bonetto \& Ezcurra de Drago, 1967)}

Spongilla (Euspongilla) schubarti Bonetto \& Ezcurra de Drago, 1967: 339-341, Lam. I figs 1, 2a,b; Holótipo: (não examinado) arroio Cuñá Pirú, provincia de Missiones, Argentina, presumivelmente depositado na coleção do Instituto Nacional de Limnologia, Santa Fé, Argentina. Parátipos: (não examinados) do arroio Cuñá Pirú, província de Missiones, Argentina; de Cataratas do Iguaçu, Missiones; de Passo de la Patria, Corrientes; do arroio Cuaró, afluente do Uruguai e de Salto Grande, rio Uruguai, também presumivelmente depositados na coleção do Instituto Nacional de Limnologia, Santa Fé, Argentina; Bonetto \& Ezcurra de Drago, 1968: 429, 430, 435; Bonetto \& Ezcurra de Drago, 1969: 353; Ezcurra de Drago \& Bonetto, 1969: 364-367, Lam II, fig. 5; Bonetto \& Ezcurra de Drago, 1970: 52, 53, 56.

Oncosclera schubarti; Volkmer-Ribeiro, 1981: 89; Matsuoka \& Masuda, 2000: 132, 134. 

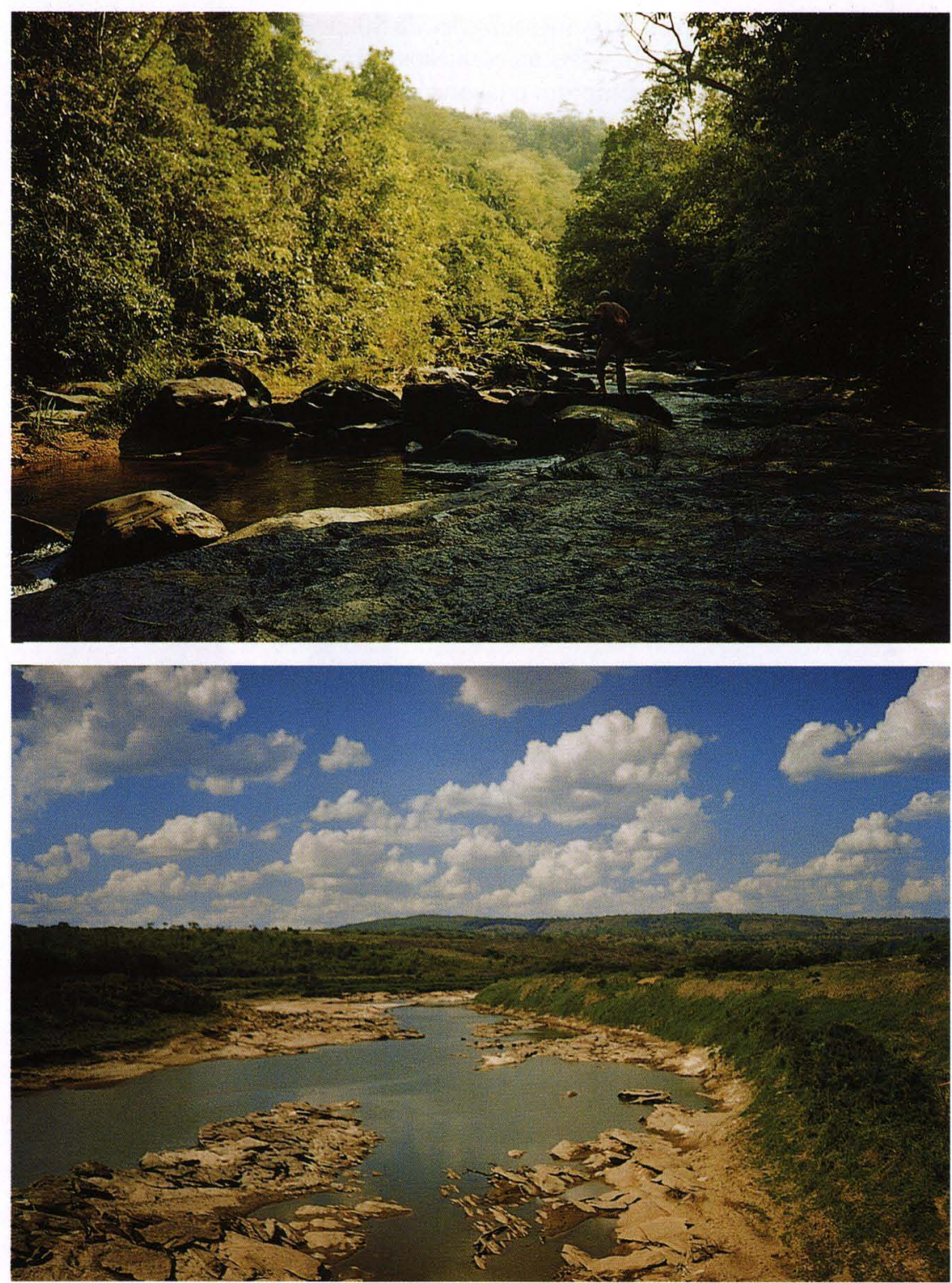

Figs 2-3. (2) Córrego do Libório evidenciando o leito constituído por rochas de grande porte; (3) Rio Corumbá à jusante da barragem da Usina Hidrelétrica Corumbá, com fluxo reduzido expondo porções do leito rochoso/arenoso normalmente encobertas. As esponjas foram encontradas constituindo manchas pequenas nas reentrâncias das rochas opostas à correnteza. Fotografias de C.V. Ribeiro.

Revta bras. Zool. 19 (1): 123 - 136, 2002 
Diagnose. Espécie de Oncosclera com gemoscleras anfiestrôngilos curtos, robustos, a maioria com porção média inflada, com superfície total ou parcialmente coberta por espinhos robustos, dispostos homogeneamente ou concentrados nas extremidades, ocorrendo ainda formas cilíndricas. Gemoscleras jovens lisas com extremidades cilíndricas afiladas, com distinção marcante entre a porção mediana inflada e as extremidades afiladas. Megascleras óxeas, de lisas a microespinhadas.

Localidade-tipo. Arroio Cuñá Pirú, província de Missiones, Argentina.

Redescrição. Esponja constituindo crostas tênues $( \pm 2 \mathrm{~mm})$ e esparsas de tamanhos variados sobre substrato rochoso. Logo acima da placa basal, que é clara, a esponja é constituída por uma camada de gêmulas sobrepostas, entre e sobre as quais se depositam de modo desordenado as gemoscleras. Acima deste estrato basal constitui-se um esqueleto cinzento denso e desordenado de megascleras que não chegam a formar feixes regulares. A superfície da esponja é lisa com orifícios osculares puntiformes. A cor dos espécimes secos varia do amarelo esbranquiçado ao cinza claro. A esponja apresenta-se ainda como grupos de gêmulas recobertas por uma película coriácea da mesma cor do substrato rochoso, aparentando saliências mamiliformes desse substrato.

Megascleras. Óxeas microespinhadas, algumas vezes lisas na porção mediana ou completamente lisas, retas a levemente curvas, delgadas a robustas com extremidades abruptas e pouco afiladas (Figs 6 e 9). Comprimento de $111 \mu \mathrm{m}$ a 421 $\mu \mathrm{m}$ (média: 191,7 $\mu \mathrm{m}$ ) e largura de $07 \mu \mathrm{m}$ a $20 \mu \mathrm{m}$ (média: 10,8 $\mu \mathrm{m}$ ).

Microscleras. Ausentes.

Gemoscleras. Anfiestrôngilos, curvos, raramente retos, progredindo de delgados a extremamente robustos, lisos a fortemente espinhados, sempre conspicuamente inflados na porção mediana e nas extremidades. As formas jovens são delgadas, completamente lisas e infladas somente na porção mediana e com seu desenvolvimento, tornam-se mais robustas e apresentam espinhos cobrindo toda a superfície da espícula, podendo estes se concentrarem nas extremidades ou estarem irregularmente distribuídos (Figs 6 e 9). As gemoscleras menores atingem formas quase esféricas, completamente cobertas por espinhos. Comprimento de $36,4 \mu \mathrm{m}$ a $125 \mu \mathrm{m}$ (média: $82,7 \mu \mathrm{m}$ ) e largura de $8 \mu \mathrm{m}$ a $24,6 \mu \mathrm{m}$ (média: $15,8 \mu \mathrm{m}$ ).

Gêmulas. Numerosas, pequenas, de esféricas a globoelípticas, forâmen provido de tubo foraminal curto (Fig. 7). A parede gemular é constituída por uma membrana interna espessa sobre a qual depositam-se de modo desordenado as gemoscleras, cobertas paulatinamente pela delgada camada externa. Camada pneumática ausente (Figs 7-8). Diâmetro de 400 a $514 \mu \mathrm{m}$ (média: 460,1 $\mu \mathrm{m}$ ).

Comentários. A descrição original não menciona local de depósito do Holótipo e Parátipos, nem o número de Parátipos.

A espécie ocorreu no rio Corumbá com grande variedade de dimensões e robustez maior que as da descrição original para os constituintes espiculares.

A redescrição oferecida acrescenta o desenvolvimento das gemoscleras a partir de anfiestrôngilos lisos e extremamente delgados (Fig. 6), a deposição das gemoscleras de modo desordenado e com quase ausência de espongina diretamente sobre a camada gemular interna, a presença de tubo foraminal e a ausência de camada pneumática. 

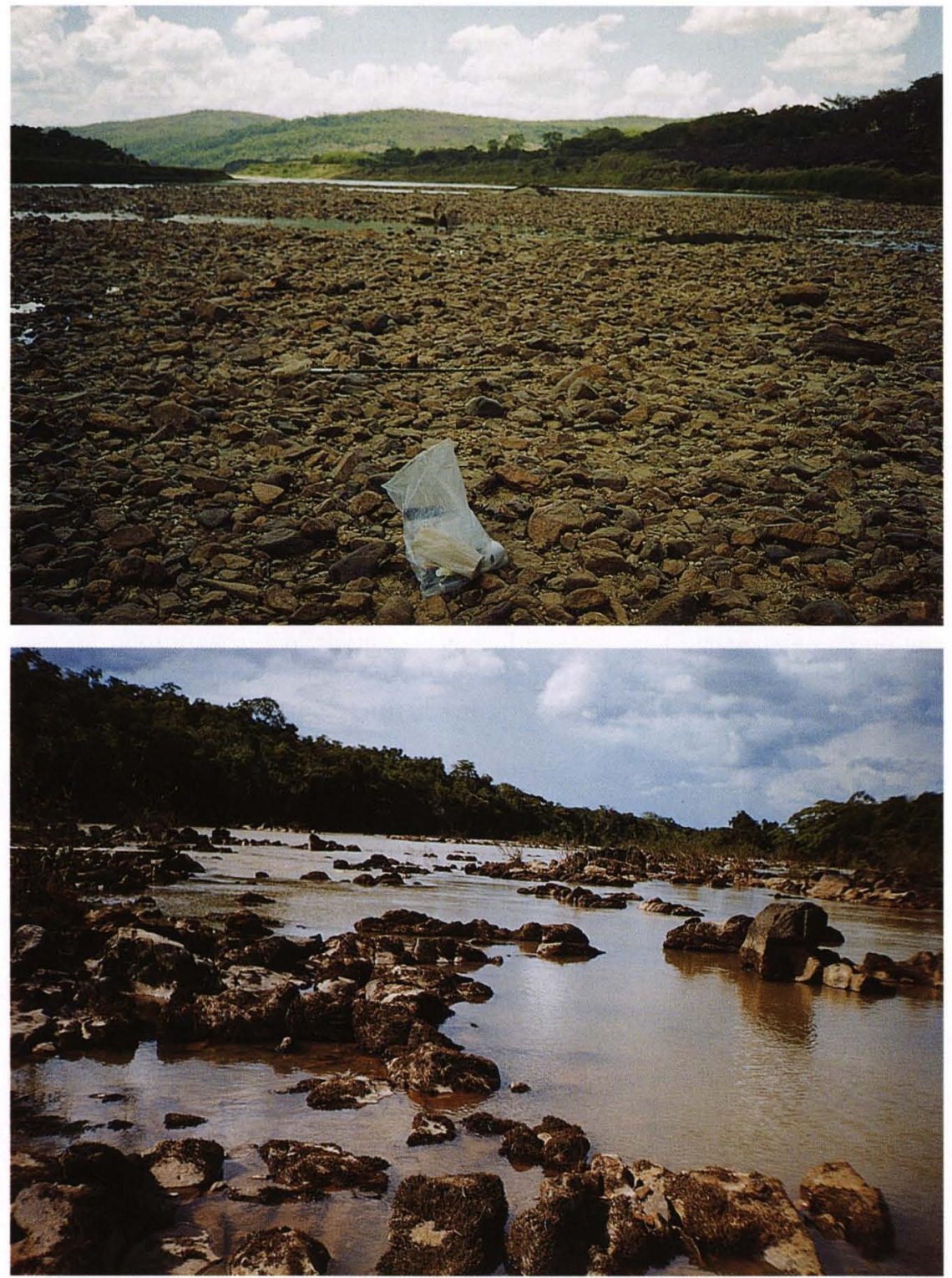

Figs 4-5 (4) Rio Corumbá à jusante da barragem da Usina Hidrelétrica Corumbá, evidenciando trecho do leito completamente exposto constituído por pedras roladas. Somente gêmulas de esponjas foram encontradas fixadas na face inferior das pedras; (5) Rio Manso à jusante da Usina Hidrelétrica de Manso, mostrando o leito rochoso irregular normalmente encoberto. As esponjas foram encontradas formando crostas pequenas nas pedras ainda cobertas por água. Fotografias de C.V. Ribeiro.

Revta bras. Zool. 19 (1): 123 - 136, 2002 


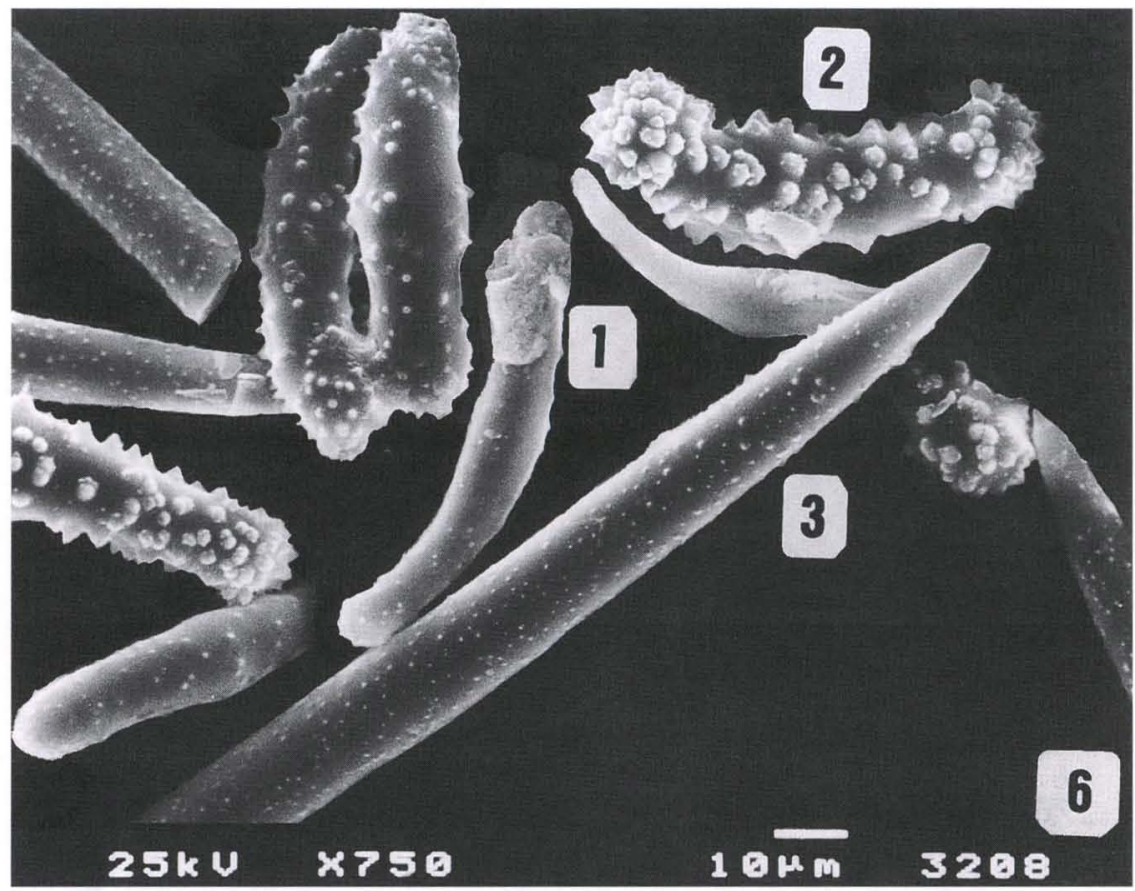

Fig. 6. Fotografia ao microscópio eletrônico de varredura (MEV) de gemoscleras jovens (1) e adultas (2) e de megascleras (3) de Oncosclera schubarti.

\section{Spongillidae Gray, 1867}

\section{Eunapius Gray, 1867}

\section{Eunapius fragilis (Leidy, 1851)}

Material examinado. BRASIL, Mato Grosso: Chapada dos Guimarães (jusante do Aproveitamento Múltiplo Manso (APM Manso), rio Manso), 30.XI.1999, C.V. Ribeiro \& C.M.C.Leite leg. (MCN 4357).

\section{Corvospongilla Annandale, 1911}

Corvospongilla seckti Bonetto \& Ezcurra de Drago, 1966

Material examinado. BRASIL, Goiás: Corumbaíba (jusante da UHE Corumbá, rio Corumbá), 04.X.1996, C.V. Ribeiro leg. (MCN 3210, 3212 e 3245); Mato Grosso: Chapada dos Guimarães (jusante da APM Manso, rio Manso), 30.XI.1999, C. Volkmer-Ribeiro \& C.M.C. Leite leg. (MCN 4360, 4362, 4368, 4369, 4370, 4381 e 4382); C. Volkmer-Ribeiro \& C.M.C. Leite leg. 01.XII.1999 (MCN 4411, 4418 e 4426). 

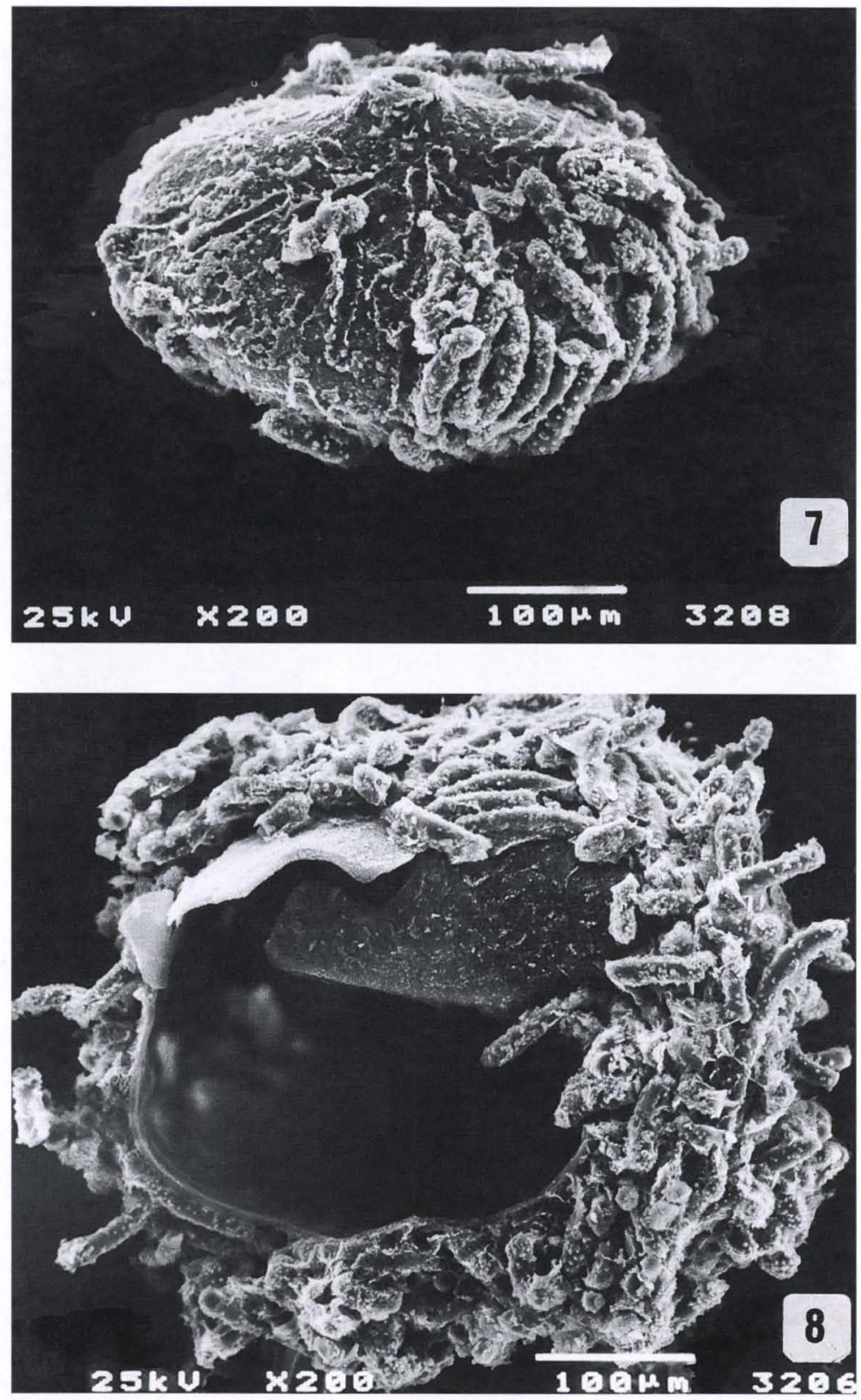

Figs 7-8. Fotografias ao microscópio eletrônico de varredura (MEV) de Oncosclera schubarti. (7) Gêmula jovem evidenciando a deposição das gemoscleras sobre a camada interna e o tubo foraminal curto; (8) gêmula adulta mostrando a camada interna grossa, ausência da camada pneumática e superposição desordenada das gemoscleras com reduzida quantidade de espongina. 


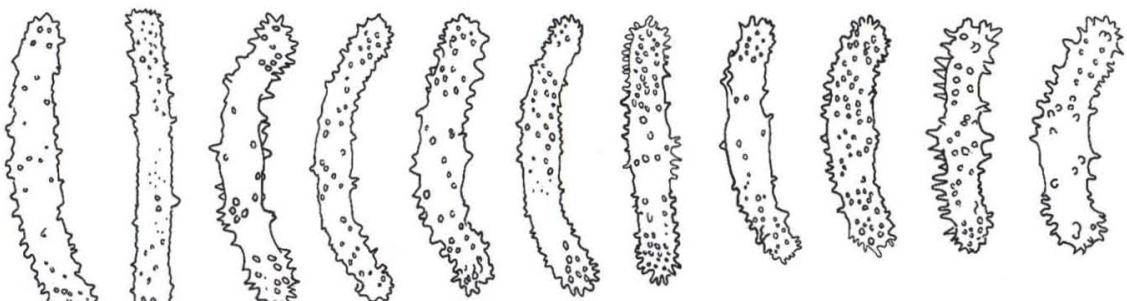

Gm
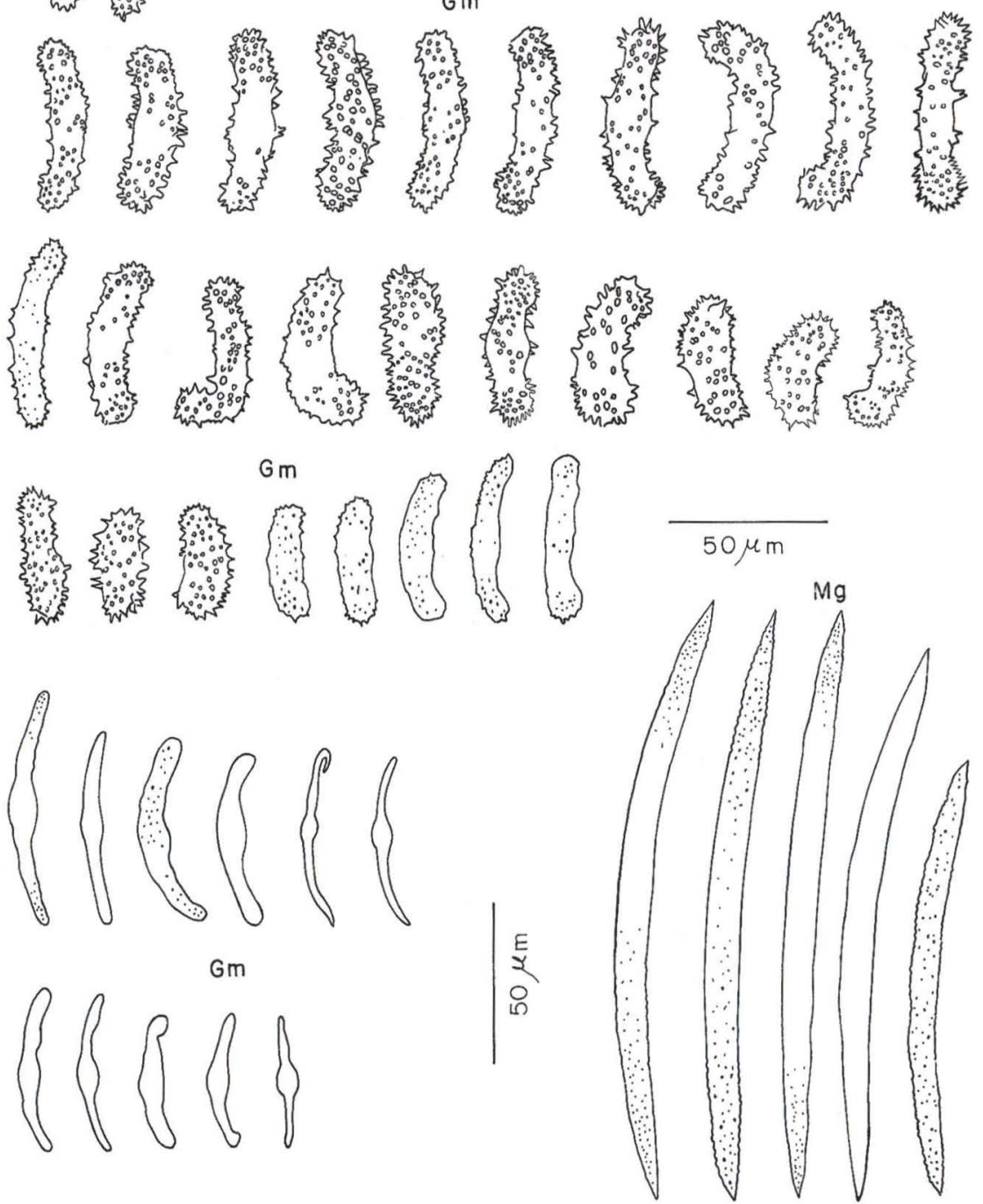

Fig. 9. Desenhos à câmara lúcida dos conjuntos espiculares de O. schubarti. (Gm) Gemoscleras, $(\mathrm{Mg})$ megascleras. Escala $50 \mu \mathrm{m}$. 


\section{Trochospongilla Vejdovsky, 1883 \\ Trochospongilla repens (Hinde, 1888)}

Material examinado. BRASIL, Goiás: Corumbaíba (jusante da UHE Corumbá, rio Corumbá), 30.IX.1996, C. Volkmer-Ribeiro leg. (MCN 3242, MCN 3246, 3257 e 3258); 04.X.1996, C. Volkmer-Ribeiro leg. (MCN 3209 e 3211); Ipamerí (montante da UHE Corumbá, córrego Santo Antônio), 02.X.1996, C. Volkmer-Ribeiro \& E. R. Alexandre leg. (MCN 3207); Mato Grosso: Chapada dos Guimarães (jusante da APM Manso, rio Manso), 30.XI.1999, C. Volkmer-Ribeiro \& C.M.C.Leite leg. (MCN 4347, 4351, 4364, 4367, 4371, 4389 e 4399); C. VolkmerRibeiro \& C.M.C.Leite leg. 01.XII. 1999 (MCN 4412, 4413, 4414, 4419 e 4422).

\section{Potamolepidae Brien, 1967}

\section{Oncosclera Volkmer-Ribeiro, 1970 \\ Oncosclera navicella (Carter, 1881)}

Material examinado. BRAsIL, Mato Grosso: Chapada dos Guimarães (jusante da APM Manso, rio Manso), 30.XI.1999, C. Volkmer-Ribeiro \& C.M.C.Leite leg. (MCN 4350, 4352, 4355, 4363, 4373, 4377 e 4398).

\section{Oncosclera schubarti (Bonetto \& Ezcurra de Drago, 1967)}

Material examinado. BRASIL, Goiás: Corumbaíba (montante da UHE Corumbá, Córrego do Libório), 01.X.1996, C. Volkmer-Ribeiro \& L.A.C. Porto leg. (MCN 3273); (jusante da UHE Corumbá, rio Corumbá), 04.X.1996, C. VolkmerRibeiro leg. (MCN 3244); Ipamerí (montante da UHE Corumbá, córrego Santo Antônio), 02.X.1996, C. Volkmer-Ribeiro \& E. R. Alexandre leg. (MCN 3206 e 3208).

\section{Oncosclera petricola (Bonetto \& Ezcurra de Drago, 1969)}

Material examinado. BRASIL, Mato Grosso: Chapada dos Guimarães (jusante da APM Manso, rio Manso), 30.XI.1999, C. Volkmer-Ribeiro \& C.M.C.Leite leg. (MCN 4348, 4353, 4356, 4365, 4366, 4374, 4378, 4380, 4387, 4395 e 4396); 01.XII.1999, C. Volkmer-Ribeiro \& C.M.C.Leite leg. (MCN 4415, 4421 e 4425); 04.XII.1999, C. Volkmer-Ribeiro \& E.C.Oliveira leg. (MCN 4431, 4433 e 4434).

Metaniidae Volkmer-Ribeiro, 1986

\section{Drulia Gray, 1867}

\section{Drulia uruguayensis Bonetto \& Ezcurra de Drago, 1968}

Material examinado. BRASIL, Goiás: Corumbaíba (montante da UHE Corumbá, Córrego do Libório), 01.X.1996, C. Volkmer-Ribeiro \& L. A. C. Porto leg. (MCN 3261 a 3268, MCN 3270 e 3271); Ipamerí (montante da UHE Corumbá, córrego Santo Antônio), 02.X.1996, C. Volkmer-Ribeiro \& E. R. Alexandre leg. 
(MCN 3204 e 3205); Mato Grosso: Chapada dos Guimarães (jusante da APM Manso, rio Manso), 30.XI.1999, C. Volkmer-Ribeiro \& C.M.C.Leite leg. (MCN 4349, 4354, 4358 e 4372); 01.XII.1999 C. Volkmer-Ribeiro \& C.M.C.Leite leg. (MCN 4402 e 4420); 04.XII.1999, C. Volkmer-Ribeiro \& E.C. Oliveira leg. (MCN 4432).

\section{DISCUSSÃO}

Os blocos rochosos ou pedras volumosas sobre os quais as esponjas estavam fixadas obrigaram destacar os espécimes do seu suporte no momento da coleta. Dada a pouca espessura dessas esponjas, as amostras consistem em fragmentos diminutos e delgados, porém, com gêmulas.

O tipo de leito apresentado pelo rio Corumbá mostrou-se completamente diferente daquele do rio Manso, devido à quantidade de areia e silte do fundo, prevendo-se uma redução da fauna de esponjas no Corumbá, conforme constatado. Nesse rio os raros espécimes de $T$. repens, $C$. seckti e $O$. schubarti coletados mostraram-se cobertos de silte e incrustavam a face superior de rochas que se projetavam do leito arenoso ou trechos do fundo cobertos por pedras irregulares e isentos de depósito de areia. Os leitos dos córregos amostrados revelaram a ocorrência de esponjas formando crostas pequenas e delgadas na parte inferior de pedras de diversos tamanhos, em ambiente de torrente. D. uruguayensis, registrada para esses córregos, é típica de tais ambientes.

A observação da tabela I mostra que, dos 26 espécimes coletados no Corumbá e nos dois córregos contribuintes, $D$. uruguayensis foi a mais abundante (46,2\% das amostras). No rio Manso, onde o número de espécimes foi mais expressivo $(\mathrm{N}=54)$, a espécie mais abundante foi $O$. petricola $(31,5 \%)$.

Tabela I. Análise quantitativa das espécies encontradas no leito dos rios Corumbá e Manso, na área das Usinas Hidrelétricas Corumbá e Manso. (N) Número de espécimes.

\begin{tabular}{|c|c|c|c|c|}
\hline \multirow{2}{*}{ Espécies } & \multicolumn{2}{|c|}{ UHE Corumbá } & \multicolumn{2}{|c|}{ UHE Manso } \\
\hline & $N=26$ & Porcentagem & $N=54$ & Porcentagem \\
\hline \multicolumn{5}{|l|}{ Spongillidae } \\
\hline Corvospongilla seckti Bonetto \& Ezcurra de Drago, 1966 & 3 & 11,5 & 10 & 18,5 \\
\hline Eunapius fragilis (Leidy, 1851) & - & - & 1 & 1,8 \\
\hline Trochospongilla repens (Hinde, 1888) & 7 & 26,9 & 12 & 22,2 \\
\hline \multicolumn{5}{|l|}{ Potamolepidae } \\
\hline Oncosclera navicella (Carter, 1881) & - & - & 7 & 13,0 \\
\hline Oncosclera petricola (Bonetto \& Ezcurra de Drago, 1969) & - & - & 17 & 31,5 \\
\hline Oncosciera schubarti (Bonetto \& Ezcurra de Drago, 1967) & 4 & 15,4 & - & - \\
\hline \multicolumn{5}{|l|}{ Metaniidae } \\
\hline Drulia uruguayensis Bonetto \& Ezcurra de Drago, 1968 & 12 & 46,2 & 7 & 13,0 \\
\hline
\end{tabular}

Acredita-se que a fauna levantada deve ter ficado aquém da realidade, uma vez que porções mais profundas do leito continuaram submersas. No entanto, o tipo de substrato encontrado e as perturbações ocasionadas no leito dos dois rios por atividades de mineração, desde o período colonial, devem ter contribuído com alguma redução da fauna. 
A comparação com as espécies levantadas por BONETTO \& EZCURRA DE DRAGO $(1967,1970)$ (Tab. II) restringiu-se àquelas que os autores citam para o Alto Paraná, na Argentina, o qual corresponde, de fato, ao curso médio continental do Paraná, enquanto as espécies que citam para o Paraná Médio são as dos lagos de planície de inundação do rio, no seu curso inferior. A fauna levantada não mostrou espécies distintas das registradas pelos supra citados autores para o Paraná Médio, à exceção de Eunapius fragilis, configurando uma comunidade típica da bacia do Paraná, estendendo-se à região centro-oeste do Brasil.

Tabela II. Relação das espécies de esponjas identificadas nos rios Corumbá e Manso, comparada com a fauna encontrada no Paraná Médio, em território Argentino (*).

Espécies

\begin{tabular}{|c|c|c|c|c|}
\hline \multicolumn{3}{|c|}{$\begin{array}{c}\text { UHE } \\
\text { Corumbá }\end{array}$} & $\begin{array}{l}\text { UHE } \\
\text { Manso }\end{array}$ & $\begin{array}{l}\text { Paraná } \\
\text { Médio (*) }\end{array}$ \\
\hline $\begin{array}{c}\text { Rio } \\
\text { Corumbá }\end{array}$ & $\begin{array}{l}\text { Córrego } \\
\text { do Libório }\end{array}$ & $\begin{array}{l}\text { Córrego Santo } \\
\text { Antônio }\left({ }^{* *}\right)\end{array}$ & $\begin{array}{c}\text { Rio } \\
\text { Manso }\end{array}$ & $\begin{array}{c}\text { Bonetto \& } \\
\text { Ezcurra de } \\
\text { Drago } \\
(1967,1970)\end{array}$ \\
\hline
\end{tabular}

\section{Spongillidae}

Anheteromeyenia ornata (Bonetto \& Ezcurra de Drago, 1970)

Corvospongilla seckti (Bonetto \& Ezcurra de Drago, 1966

Eunapius fragilis (Leidy, 1851)

Houssayella iguazuensis Bonetto \& Ezcurra de Drago, 1966

Trochospongilla repens (Hinde, 1888)

Trochospongilla paulula (Bowerbank, 1863)

Potamolepidae

Oncosclera atrata Bonetto \& Ezcurra de Drago, 1970

Oncosclera navicella (Carter, 1881)

Oncosclera petricola (Bonetto \& Ezcurra de Drago, 1969)

Oncosclera ponsi Bonetto \& Ezcurra de Drago, 1968

Oncosclera schubarti (Bonetto \& Ezcurra de Drago, 1967)

Oncosclera stolonifera Bonetto \& Ezcurra de Drago, 1967

Metaniidae

Drulia browni (Bowerbank, 1863)

Drulia uruguayensis Bonetto \& Ezcurra de Drago, 1968

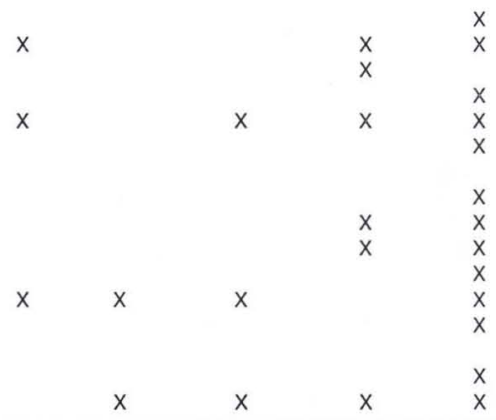

(*) As citações de BonetTo \& EzcurRA de Drago $(1967,1970)$ para o Paraná Médio obedecem às sinonímias propostas por VOLKMER-RIBEIRO (1981), VolKMER-RIBEIRO \& DE ROSA-BARBOSA (1985) e VOLKMER-RIBEIRO \& TAVARES (1995), para respectivamente as espécies dos gêneros Oncosclera, Trochospongilla e Drulia.

$\left.{ }^{* *}\right)$ dados abióticos: temperatura da água $23,5^{\circ} \mathrm{C}, \mathrm{pH} 6,8, \mathrm{O}_{2}$ dissolvido $8,5 \mathrm{mg} / \mathrm{l}$ e condutividade $21,4 \mu \mathrm{S} / \mathrm{cm}$.

O estudo das novas amostras possibilitou a redescrição de $O$. schubarti, confirmando seu status específico e, sobretudo, sua inclusão no gênero Oncosclera como proposto por VOLKMER-RIBEIRO (1981). As novas características reveladas na formação das gêmulas de $O$. schubarti confirmam aspectos descritos por BRIEN (1970), para a formação das mesmas em Potamolepídeos da região Etiópica. Quaisquer novas características descritas para gêneros e espécies de Potamolepidae revestem-se agora de nova importância dado o recente registro de espécie fóssil de Oncosclera no Mioceno do Japão (MATSUOKA \& MASUDA 2000).

O levantamento realizado estabelece subsídios para o acompanhamento da eventual colonização do lago por essa fauna. Registros de colonização por esponjas em lagos de Usinas hidrelétricas foram apresentados por VOLKMER-RIBEIRO \&

Revta bras. Zool. 19 (1): 123 - 136, 2002 
HATANAKA (1991), com a colonização do lago da UHE Tucuruí (PA) por $O$. spinifera Bonetto \& Ezcurra de Drago, 1973 e C. seckti, sendo também evidenciadas à juzante da barragem uma comunidade de esponjas de fundo rochoso constituída por $D$. uruguayensis, $O$. navicella, $T$. repens e $C$. seckti. VOLKMER-RIBEIRO \& LOPES DE CARVALHO (in litt.) registraram, após 15 anos da sua formação, a ocupação do lago da UHE Curuá-Una (PA) por duas espécies de esponjas, Spongilla spoliata Volkmer-Ribeiro \& Maciel, 1983 e O. intermedia (Bonetto \& Ezcurra de Drago, 1973), sendo a primeira dominante, acredita-se devido a uma maior produção tanto de gêmulas quanto de larvas.

AGRADECIMENTOS. A Furnas S.A., pelo convite para participação nas Operações de Salvamento de Fauna e pelo apoio logístico prestado, particularmente ao biólogo Hamilton Garboggini pela gentileza das diversas solicitações atendidas. Aos coordenadores das Operações de Salvamento de Fauna, biólogo Hélder Lúcio Rodrigues da Silva e Prof. Dr. Nelson Jorge da Silva Jr. (UHE Corumbá), Prof. Dr. Cleber J.R. Alho e M.Sc. Luiz Augusto S. Vasconcelos (UHE Manso) pela solicitude constante e apoio nas atividades de prospecção. Ao M.Sc. Luiz Augusto da Costa Porto pelos dados abióticos constantes na tabela II. À M.Sc. Karin M. Grosser, M.Sc. Laura M.G. Tavares e à desenhista Rejane Rosa, MCN/FZB por respectivamente: dados de localização (GPS), colaboração na obtenção das fotos ao MEV e arte final dos desenhos das espículas.

\section{REFERÊNCIAS BIBLIOGRÁFICAS}

Bonetto, A.A. \& I. Ezcurra de Drago. 1967. Esponjas del noreste Argentino. Acta Zool. Lilloana.

23: 331-348. (75): $429-436$.

. 1969. “Notas sistematicas sobre el genero Uruguaya Carter (Porifera, Spongillidae)”. Physis. 28 (77): 351-357.

- 1970. Esponjas de los afluentes del Alto Parana en la Provincia de Missiones. Acta Zool. Lilloana. 27: 37-58.

BrIEN, P. 1970. Les Potamolepides Africaines Nouvelles du Luapula et du lac Moero. Symp. Zool. Soc. Lond. 25: 163-187.

Ezcurra de Drago, I. \& A.A. Bonetto. 1969. Algunas caracteristicas del bentos en los saltos del rio Uruguay, con especial referencia a la ecologia de los Poriferos. Physis 28 (77): 359-369.

MatsuoKa, K. \& Y. Masuda. 2000. A new potamolepid freshwater sponge (Demospongiae) from the Miocene Nakamura Formation, central Japan. Paleontol. Res. 04 (02): 131-137.

Volkmer-Ribeiro, C. 1981. Porifera, p. 86-95. In: S.H. Hurlbert; G. Rodriguez \& N.D. Santos (Eds). Aquatic Biota of Tropical South America. Part 2: Anartropoda. San Diego, San Diego State Univ., XI+298p.

- 1985. Manual de Técnicas para a preparação de Coleções Zoológicas 3. São Paulo, Sociedade Brasileira de Zoologia, CNPq, 6p.

Volkmer-Ribeiro, C. \& R. DE Rosa-Barbosa. 1978. A new genus and species of Neotropical freshwater sponges. Iheringia, Sér. Zool., Porto Alegre, (52): 103-107.

. 1985. Redescription of the freshwater sponges Trochospongilla repens (Hinde, 1888) and Trochospongilla amazonica (Weltner, 1895) with an account of the South American species of Trochospongilla (Porifera, Spongillidae). Iheringia, Sér. Zool., Porto Alegre, (65): 77-93.

Volkmer-Ribeiro, C. \& T. HATANAKA. 1991.Composição específica e habitat da espongofauna no lago da UHE de Tucuruí, PA). Iheringia, Sér. Zool., Porto Alegre, (71): 177-178. 
Volkmer-Ribeiro, C. \& J.F.M. MotTA. 1995. Esponjas formadoras de espongilitos em lagoas do triângulo mineiro e adjacências, com indicação de preservação de habitat. Biociências, Porto Alegre, 3 (2): 145-69.

Volkmer-Ribeiro, C. \& M.C.M. Tavares. 1995. Redescrição de Drulia uruguayensis Bonetto \& Ezcurra de Drago, 1968 com redefinição do gênero Drulia Gray, 1867 (Porifera: Metaniidae). Biociências, Porto Alegre, 3 (1): 183-205.

VolKmER-Ribeiro, C. \& B. TURCQ. 1996. SEM analysis of silicious spicules of a freshwater sponge indicate paleoenvironmental changes. Acta Microscopica 5 (B): 186-7.

Recebido em 19.X.2000; aceito em 04.II.2002. 